\title{
Who cares for the adult brain damaged? Hamble Ward revisited
}

\author{
Charles R. Shawcross, Consultant Psychiatrist (correspondence); John Crowder, \\ Ward Manager; KAREN BROWNRIGG, Senior Occupational Therapist; Allan READ, \\ Senior Clinical Psychologist; JANe STREet, Psychology Technician; Jill WatkIns, \\ Social Worker; and VAL POMEROY, Senior Physiotherapist, Knowle Hospital, \\ Fareham, Hants PO17 5NA
}

In 1983, Dr Newson-Smith wrote an article in the Bulletin on services for the younger brain damaged persons. In particular she described Hamble Ward, which provides care for such people and for which she had medical responsibility. At that time, this type of service was very rare in the UK and the article generated considerable interest. In 1984, Dr Newson-Smith left the hospital and was replaced by one of the authors (CRS).

Since 1984, many other Districts have set up, or are in the process of setting up similar services, and some private nursing homes are taking on this role more formally. Our own service has changed considerably over the past six years and we thought that it may be helpful if we updated Dr Newson-Smith's article.

\section{Hamble Ward}

Hamble Ward is a 16-bedded unit based at Knowle Hospital, Hants. The patients on the ward suffer from a range of disorders, but these can be roughly divided into the following groups: those with slowly progressive neurological disorders, e.g. Huntington's Disease; those with severe but nonprogressive neurological deficits who will probably require care in a hospital setting for the rest of their lives; and those who are slow rehabilitation prospects. The ward also caters for a certain number of day cases. The multidisciplinary team includes members from all the major mental health professions including physiotherapy and speech therapy.

\section{Hamble Ward Assessment Profile}

In general psychiatric work, the fact that someone has not, for example, gone shopping recently, may not be viewed as a problem, and yet for people with a long-term disorder such activities often never take place without well organised help. In a busy ward round, these issues may not be covered. As an attempt to remedy this failing we designed a new assessment profile. The Hamble Ward Assessment
Profile was partly inspired by the idea of Life Planning (Chamberlain, 1985) which was originally developed for people with mental handicaps with the aim of helping them to live an ordinary life while acknowledging their need for a great deal of help if this is to be achieved (King's Fund, 1980).

The Hamble Ward Assessment requires succinct statements of need from each profession following their own investigation of a patient. This includes, for example, details from nurses about a typical day in the patient's life and the help that might be needed to carry out a wide variety of activities of daily living.

At the case review, this information is read out and all areas of need are considered, not forgetting ordinary needs such as decent clothes, more frequent contact with a friend, something to do during the day, etc. Action is then agreed as to how to begin meeting these needs and which member or members of the team will be responsible. If needs cannot be met, this is recorded.

\section{Computerised day}

On a busy ward, with relatively low staffing levels and many patients with very high levels of dependency, it is easy for the less 'necessary' tasks, such as taking someone out for a pub lunch, to be pushed out by more pressing demands such as toileting. If staff are not careful these essential tasks may be the only ones that get carried out. It can easily happen, that a carefully worked-out comprehensive package of interventions written into the assessment profile is largely ignored. To try and overcome this concern we decided to introduce the 'computerised day'.

The 'computerised day' consists of sheets, placed on the office wall, one for each day of the week. On these sheets, there is one column for the times of day, and one for the activities scheduled including the names of the patients involved. There is a further column to indicate whether activities have been performed, and if not, the reason is to be indicated. The sheets are produced on a word processor, and are 
updated each week to incorporate any changes made at the weekly care reviews.

This development appears to have been very successful in increasing the number of programmed activities patients perform, and enables the team to see at a glance when interventions can be reasonably timed, and when staffing will make a suggestion unworkable.

\section{Structured day}

When not engaged in specific therapies, residents should be offered the opportunity to engage in a wide range of activities, from watching television to going for a swim. It is not that these activities should be forced upon them for their 'own good', but more that they should at least be offered. Too often the option of watching television can take precedence over other possibly preferable pastimes.

In an effort to overcome this problem, a multidisciplinary structured day project was begun. Nurses give patients a choice of ordinary life activities, e.g. playing games, going out, preparing food, listening to music, coffee mornings in small groups, etc., and record who engages in which activity. In this way a crucial feature of a patient's quality of life - "what does he do all day?" - is monitored and the evaluation is fed back to the entire ward team. Patients have been designated to keyworkers in small groups so that they can experience a structured day together.

\section{Other developments}

In the last two years, we have started a 'Friends of Hamble Ward Association'. This group meets once a month in the evening and provides an opportunity for friends, relatives and staff to talk in an informal atmosphere. The 'Friends' have arranged many functions for the residents and raised large amounts of money for the ward. We have also introduced a fortnightly community meeting where residents can make their feelings known. We have been pleasantly surprised at the extent to which these very damaged people are able to articulate their views.

\section{Comment}

We would not wish to create the impression that we have found the ideal way of running a Brain Damage Unit, but the changes that we have introduced have had an observably beneficial effect. The information is in a form suitable for more formal analysis as part of medical audit, or in making a case for increased resources.

\section{Acknowledgements}

We wish to thank Dr M Ledger and Ms I Clarke for their help in developing the 'computerised day'.

\section{References}

Chamberlain, P. (1985) Life Planning Manual. British Association for Behavioural Psychotherapy, 59 Revelstoke Road, Wimbledon Park, London SW18 5NJ.

KING's Fund (1980) An Ordinary Life (Comprehensive Locally Based Residential Services for Mentally Handicapped People). Project Paper No. 24. London: King's Fund Centre.

NeWSON-SmITH, J. (1983) Who cares for the adult brain damaged? Bulletin of the Royal College of Psychiatrists, 7 , 180-183. 